\section{Emergency Open Cardiac Massage via Subxyphoid Approach in Ruptured Type A Aortic Dissection}

\author{
HSING-LIN LIN • WEI-CHE LEE • SHING-GHI LIN • LIANG-CHI KUO • \\ YUAN-CHIA CHENG • CHAO-WEN CHEN
}

CHAO-WEN CHEN ( $\square$ ) Department of Trauma Kaohsiung Medical University Hospita 100 Tzyou 1st Road Kaohsiung 807, Taiwan Phone: + 88673125895 ext 7553 Fax: +88673208255 E-mail: ytljwc@yahoo.com.tw

HSING-LIN LIN • WEI-CHE LEE - SHING-GHI LIN • LIANG-CHI KUO • YUAN-CHIA CHENG Department of Trauma Kaohsiung Medical University Hospital Kaohsiung Medical University Kaohsiung, Taiwan

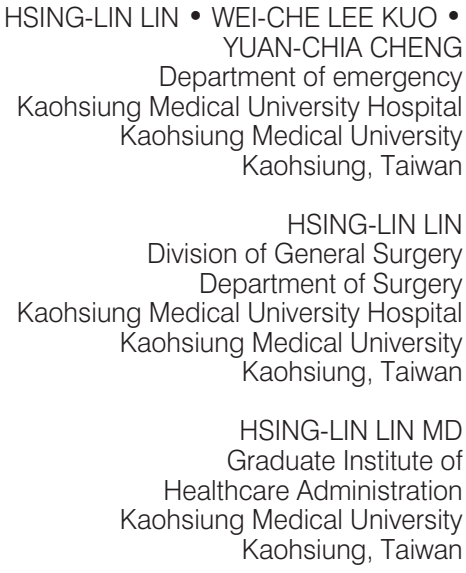

HSING-LIN LIN MD Graduate Institute of Healthcare Administration Kaohsiung Medical University Kaohsiung, Taiwan

\begin{abstract}
Patient sustained cardiac tamponade caused by rupture of type $A$ aortic dissection may result in sudden death. Pericardiocentesis is a lifesaving procedure; nevertheless, blood may occlude the catheter and fail to relieve the pressure. However, open-chest cardiac massage in resuscitation has been studied in animal models by some medical centers and laboratories with inspiring results. We report a 58-year-old woman who was transferred from a local hospital with the diagnosis of cardiac tamponade caused by ruptured type A aortic dissection. Pulseless electrical activity followed by cardiac arrest occurred thereafter. Successful resuscitation in the emergency department was achieved using open cardiac massage through the sub-xyphoid region by opening a pericardial window. Therefore, in unstable patients with cardiac tamponade due to aortic dissection, this resuscitative procedure is feasible, safe and efficient.
\end{abstract}

Keywords: direct cardiac compression, resuscitation, sub-xyphoid approach, cardiac tamponade, aortic dissection

\section{Introduction}

Patients who have aortic dissection are at great risk of rupture, which may cause cardiac tamponade and result in sudden death. Traditionally, cardiocentesis was performed to relieve the pressure in the pericardial sac, especially by physicians without specialty training in cardiothoracic surgery. However, inserting a pericardiocentesis needle may cause unexpected heart injury and the clotted blood may occlude the draining catheter. Open thoracotomy is not routinely used in the resuscitation of patients sustaining cardiac tamponade owing to the complex nature of the procedure. Nevertheless, in a report by Yanagawa $Y$ et al. a patient with lateral thoracotomy was resuscitated successfully after suddenly collapsing. (1) The benefit of open thoracotomy is that it provides not only cardiac tamponade relief but also enables direct cardiac compression. We report a patient with a similar situation who was also successfully resuscitated employing an alternative method to thoracotomy. We resuscitated the patient through the open chest via the sub-xyphoid region using pressure relief following direct cardiac massage. Comparing 
to the traditional median sternotomy, this is an early way to approach the heart, and provides a better and easier method to relieve hemorrhagic cardiac tamponade.

\section{Case report}

A 58-year-old woman presented to a local emergency department (ED) with reported altered mental status after blacking out, falling down, and receiving a head injury. According to reports from her family, she awoke soon after the blackout and complained about head and chest pain. Her family denied any previous trauma, toxic ingestions, fevers, chills, rashes, recent illnesses, or respiratory, cardiac, genitourinary, or gastrointestinal complaints. The patient had a medical history of hypertension. After arrival at the ED of a local hospital, her vital signs were stable, with a temperature of $36.6^{\circ} \mathrm{C}$, blood pressure of $114 / 65 \mathrm{~mm} \mathrm{Hg}$, pulse of 100 beats per minute, and a respiratory rate of 26 breaths per minute. Brain-computed tomography (CT) was obtained and there was no intracranial lesion. She felt chest pain and dyspnea during the ED observation. A chest radiograph was performed with the finding of mediastinal widening (figure 1a). A chest CT was then performed and Type A aortic dissection was uncovered with a massive pericardial effusion (figures $1 \mathrm{~b}$ and 1c). Due to lack of backup of cardiothoracic surgery facilities, she was soon transferred to our hospital with the diagnosis of ruptured type A aortic dissection with hemopericardium.

Upon her arrival at the ED, rapid assessment was initiated, she was found to have a patent airway, and breathing sounds were equal bilaterally. Heart sounds diminished, and pulses were weak bilaterally. Her vital signs were notable for a blood pressure of 66/45 $\mathrm{mmHg}$, and a heart rate of $96 \mathrm{bpm}$. Despite rapid resuscitation, the patient became unconscious within 10 minutes of arrival. Moreover, her blood pressure suddenly dropped and cardiac monitoring revealed pulseless electrical activity. Advanced Cardiac Life Support (ACLS) guidelines were followed with

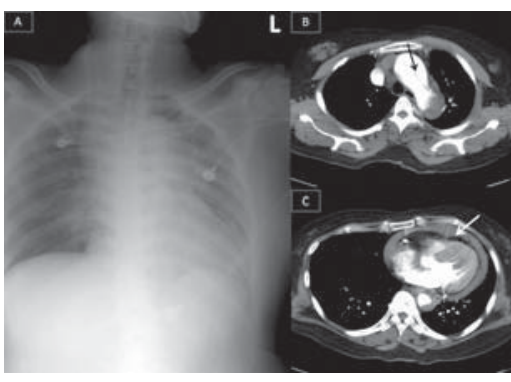

Figure 1a. The chest radiograph shows widening of the superior mediastinum.

Figure $1 \mathrm{~b}$ and 1c. Chest CT scans shows rupture of the aortic dissection with false, true lumens and an intimal flap (black arrow). Heterogeneous hyperattenuating fluid collected in the pericardium as indicated by the white arrow.

rapid endotracheal intubation. Once the endotracheal tube was successfully placed and the position confirmed, needle pericardiocentesis was performed with fresh blood drawn. However, her heart remained in asystole without any response after the drainage procedure and cardiopulmonary resuscitation (CPR). With the aim of rapid evacuation of pericardial blood, the subxyphoid pericardial window was then opened (figure 2). An eight $\mathrm{cm}$ medial incision just caudal to the xyphoid process was made. With preservation of the xyphoid process, finger dissection was made along the retrosternal space. After approaching the pericardium, a three $\mathrm{cm}$ opening in the pericardium was created for blood drainage. By placing a hand over the infra-posterior aspect of the heart as well as the other hand on the sternum, a combination of closed and open cardiac massage was performed in this manner (figure 2d). The resuscitative procedure lasted for approximately 15 minutes with several intravenous injections of epinephrine till signs of life including palpable pulse and reactive pupils were identified. With the assistance of an in-house cardiothoracic surgeon, she was rapidly sent to the operating room and the aorta was repaired along with the extended wound created at the ED.

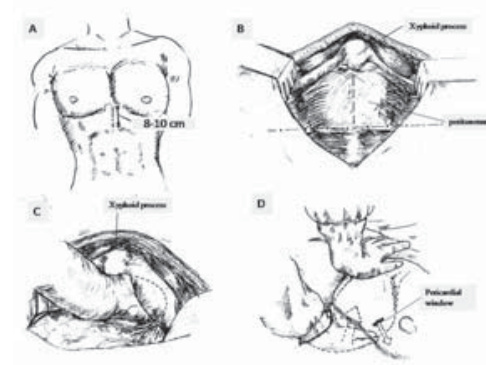

Figure 2a. A midline skin incision about $8-10 \mathrm{~cm}$ is made below the xyphoid process.

Figure 2b. The pre-peritoneum space is dissected without opening the peritoneum.

Figure 2c. Blunt dissection by fingers is used to separate the tissue under the sternum.

Figure 2d. A pericardial window is made (hollow arrow) prior to cardiac compression between one hand on the sternum and the other hand on the infra-posterior aspect of the heart.

\section{Discussion}

Open cardiac massage has been found to be superior than closed chest compressions as indicated by the doubledup cardiac index and coronary perfusion pressure. (2) In the past, open-chest cardiopulmonary resuscitation was limited in the emergency room at level I trauma centers and performed especially in traumatic cardiac arrest patients. (3-6) Although there is still controversy over open-chest CPR being used in clinical practice to revive the non-traumatic cardiac arrest victim, (7) there are still sporadic reports about successful resuscitation with direct open chest cardiac compression in cardiac tamponade. (1)

Most physicians do not have sufficient confidence to perform resuscitative thoracotomy due to lack of experience. Advanced Trauma Life Support ${ }^{\circledR}$ also claims qualified surgeons are essential while making the decision for resuscitative thoracotomy. Conventionally, antero-lateral thoracotomy is used to achieve good exposure and bleeding control of intra-thoracic injuries. Howe- 
ver, without the aid of a rib retractor, one cannot perform cardiac massage easily through the narrow intercostal space. Alternatively, the sub-xyphoid approach provides preferable access. The midline sub-xyphoid incision is easily marked and made. With particular attention to avoiding diaphragmatic injury, we can insert a whole hand into the retrosternal space without encountering overt anatomical obstacles. In addition, with direct palpation of the pericardium, a decompressive window can be made simultaneously for more effective evacuation of pericardiac effusion than closed pericardiocentesis. This procedure can be accomplished within a shorter time than antero-lateral thoracotomy and sternotomy. In such a setting as our case with aortic dissection, most cardiothoracic surgeons would perform midline sternotomy for definitive vascular surgery. The sub- xyphoid approach would not create another wound compared with conventional anterolateral thoracotomy. This manner is indeed of benefit for decreasing morbidity and promoting recovery. In our patient, we also performed a combination of open and closed cardiac massage as shown in figure 2. Open cardiac massage has been proven to be more effective than the closed method in each cardiac stroke. (2) In openchest cardiac resuscitation, approached via the anterolateral aspect, one can perform the massage by putting the heart between the palms to avoid squeezing or crushing injuries to the heart by the fingers. Nevertheless, via the sub-xyphoid approach, we propose the same caution by simultaneous compression over the infra-posterior aspect of the heart and on the sternum. Although it seemed to be effective in our case, the actual effect of this met- hod has not been discussed before. Further studies are necessary.

Although direct cardiac compression is still continuously studied in animal models, (7-10) we suggest that it not only provides better prognosis in traumatic cardiac arrest patients but also in those with cardiac tamponade caused by medical problems. Since hemorrhagic cardiac tamponade caused by a traumatic or nontraumatic ruptured aorta usually endangers vitals within a few minutes, decompression of the pericardial sac should be performed promptly and effectively. In our patient, we provided a feasible method to approach the heart as well as open the pericardium. Open cardiac massage via the subxyphoid approach might be proposed as the initial resuscitative procedure for pericardial tamponade caused by nontraumatic rupture of the aorta in addition to acute thoracic trauma.

\section{REFERENCES}

1. Yanagawa Y, Morita K, Sakamoto T, Okada Y, Isoda S, Maehara T. A satisfactory recovery after emergency direct cardiac massage in type A acute aortic dissection with cardiac arrest. Am J Emerg Med 2006;24(3):356-7.

2. Twomey D, Das M, Subramanian H, Dunning J. Is internal massage superior to external massage for patients suffering a cardiac arrest after cardiac surgery? Interact Cardiovasc Thorac Surg 2008;7(1):151-6.

3. Fialka C, Sebok C, Kemetzhofer P, Kwasny O, Sterz F, Vecsei V. Open-chest cardiopulmonary resuscitation after cardiac arrest in cases of blunt chest or abdominal trauma: a consecutive series of 38 cases. J Trauma 2004;57(4):809-14.

4. Grove CA, Lemmon G, Anderson G, McCarthy M. Emergency thoracotomy: appropriate use in the resuscitation of trauma patients. Am Surg 2002;68(4):313-6; discussion 6-7.

5. Soreide K, Soiland H, Lossius HM, Vetrhus M, Soreide JA, Soreide E. Resuscitative emergency thoracotomy in a Scandinavian trauma hospital--is it justified? Injury 2007;38(1):34-42.

6. Frezza EE, Mezghebe H. Is 30 minutes the golden period to perform emergency room thoratomy (ERT) in penetrating chest injuries? J Cardiovasc Surg (Torino) 1999;40(1):147-51.

7. Benson DM, O'Neil B, Kakish E, Erpelding J, Alousi S, Mason R, et al. Open-chest CPR improves survival and neurologic outcome following cardiac arrest. Resuscitation 2005;64(2):209-17.

8. Kern KB, Sanders AB, Badylak SF, Janas W, Carter AB, Tacker WA, et al. Long-term survival with open-chest cardiac massage after ineffective closed-chest compression in a canine preparation. Circulation 1987;75(2):498-503.

9. Kern KB, Sanders AB, Janas W, Nelson JR, Badylak SF, Babbs CF, et al. Limitations of open-chest cardiac massage after prolonged, untreated cardiac arrest in dogs. Ann Emerg Med 1991;20(7):761-7.

10. Salcido DD, Menegazzi JJ, Rittenberger JC. Electrophysiology and hemodynamics of open chest resuscitation from cardiac arrest in a swine. Acad Emerg Med 2009;16(1):89-90. 